\title{
Publisher's Note: Emulating the one-dimensional Fermi-Hubbard model by a double chain of qubits [Phys. Rev. A 94, 032338 (2016)]
}

\author{
Jan-Michael Reiner, Michael Marthaler, Jochen Braumüller, Martin Weides, and Gerd Schön
}

(2) (Received 14 February 2018; published 21 February 2018)

DOI: 10.1103/PhysRevA.97.029904

This paper was published online on 30 September 2016 with missing support information in the Acknowledgment section on page 5. On page 5, the second sentence of the Acknowledgments should read as "This work was supported by European Research Council (ERC) within consolidator Grant No. 648011, Deutsche Forschungsgemeinschaft (DFG) within Project No. WE4359/7-1." The Acknowledgments have been corrected as of 15 February 2018. The Acknowledgments are incorrect in the printed version of the journal. 